\title{
On C-Learnability in Description Logics
}

\author{
Ali Rezaei Divroodi ${ }^{1}$, Quang-Thuy $\mathrm{Ha}^{2}$, \\ Linh Anh Nguyen ${ }^{1}$, and Hung Son Nguyen ${ }^{1}$ \\ 1 Faculty of Mathematics, Informatics and Mechanics, \\ University of Warsaw, Banacha 2, 02-097 Warsaw, Poland \\ $\{$ rezaei, nguyen, son $\}$ @mimuw .edu.pl \\ 2 Faculty of Information Technology, College of Technology, \\ Vietnam National University, 144 Xuan Thuy, Hanoi, Vietnam \\ thuyhq@vnu.edu.vn
}

\begin{abstract}
We prove that any concept in any description logic that extends $\mathcal{A L C}$ with some features amongst $I$ (inverse), $Q_{k}$ (quantified number restrictions with numbers bounded by a constant $k$ ), Self (local reflexivity of a role) can be learnt if the training information system is good enough. That is, there exists a learning algorithm such that, for every concept $C$ of those logics, there exists a training information system consistent with $C$ such that applying the learning algorithm to the system results in a concept equivalent to $C$.
\end{abstract}

\section{Introduction}

Description logics (DLs) are a family of formal languages suitable for representing and reasoning about terminological knowledge [1. They are of particular importance in providing a logical formalism for ontologies and the Semantic Web. Binary classification in the context of DLs is called concept learning, as the function to be learnt is expected to be characterizable by a concept. This differs from the traditional setting in that objects are described not only by attributes but also by relationship between the objects (i.e., by object roles).

Concept learning in DLs has been studied in a considerable number of works (e.g., 3 2 8 7 5 10 11,6]). The work [3] is based on "least common subsumers", the works 2 28775 is based on refinement operators as in inductive logic programming, and the works 10116 is based on bisimulation in DLs.

PAC-learning (probably approximately correct learning) is a framework for mathematical analysis of machine learning proposed in 1984 by Valiant [12]. In this framework, the learner receives samples and must select from a certain class a hypothesis that approximates the function to be learnt. The goal is that, with high probability, the selected hypothesis will have low generalization error. The learner must be able to learn the concept in polynomial time given any arbitrary approximation ratio, probability of success, or distribution of the samples. PAClearnability is an important notion for practical learning algorithms. However, it is hard to investigate for DLs. We are aware of only the work [3] by Cohen and Hirsh, which shows PAC-learnability for a very restricted DL called C-CLASSIC. 
In this paper, we study C-learnability (possibility of correct learning) in DLs. We prove that any concept in any description logic that extends the basic DL $\mathcal{A L C}$ with some features amongst $I$ (inverse), $Q_{k}$ (quantified number restrictions with numbers bounded by a constant $k$ ), Self (local reflexivity of a role) can be learnt if the training information system is good enough. That is, there exists a learning algorithm such that, for every concept $C$ of those logics, there exists a training information system consistent with $C$ such that applying the learning algorithm to the system results in a concept equivalent to $C$.

Although C-learnability is somehow weaker than PAC-learnability, our theoretical result on C-learnability is still significant for the learning theory in DLs. Our investigation uses bounded bisimulation in DLs and a new version of the algorithms proposed in [1011]6 that minimizes modal depths of resulting concepts. It shows a good property of the bisimulation-based concept learning method proposed in 10116].

The rest of this paper is structured as follows. In Section 2 we introduce notation and semantics of DLs. In Section 3 we present concept normalization and introduce universal interpretations. In Section 4 we define bounded bisimulation in DLs and state its properties. In Section 5 we present a concept learning algorithm, which is used in Section 6 for analyzing C-learnability in DLs. Concluding remarks are given in Section 7

\section{Notation and Semantics of Description Logics}

A $D L$-signature is a set $\Sigma=\Sigma_{I} \cup \Sigma_{C} \cup \Sigma_{R}$, where $\Sigma_{I}$ is a finite set of individual names, $\Sigma_{C}$ is a finite set of concept names, and $\Sigma_{R}$ is a finite set of role names. Concept names are unary predicates, while role names are binary predicates. We denote concept names by capital letters like $A$ and $B$, role names by lower-case letters like $r$ and $s$, and individual names by lower-case letters like $a$ and $b$.

We will consider $D L$-features denoted by $I$ (inverse), $Q_{k}$ (quantified number restrictions with numbers bounded by a constant $k$ ) and Self (local reflexivity of a role). In this paper, by a set of $D L$-features we mean an empty set or a set consisting of some of these names.

Let $\Sigma$ be a DL-signature and $\Phi$ be a set of DL-features. Let $\mathcal{L}$ stand for $\mathcal{A L C}$, which is the name of a basic DL. (We treat $\mathcal{L}$ as a language, but not a logic.) The DL language $\mathcal{L}_{\Sigma, \Phi}$ allows roles and concepts defined recursively as follows:

- if $r \in \Sigma_{R}$ then $r$ is role of $\mathcal{L}_{\Sigma, \Phi}$

- if $I \in \Phi$ then $r^{-}$is a role of $\mathcal{L}_{\Sigma, \Phi}$

- if $A \in \Sigma_{C}$ then $A$ is concept of $\mathcal{L}_{\Sigma, \Phi}$

- if $C$ and $D$ are concepts of $\mathcal{L}_{\Sigma, \Phi}, R$ is a role of $\mathcal{L}_{\Sigma, \Phi}, r \in \Sigma_{R}$, and $h, k$ are natural numbers then

- $\top, \perp, \neg C, C \sqcap D, C \sqcup D, \forall R . C$ and $\exists R . C$ are concepts of $\mathcal{L}_{\Sigma, \Phi}$

- if $Q_{k} \in \Phi$ and $h \leq k$ then $\geq h R$.C and $<h R . C$ are concepts of $\mathcal{L}_{\Sigma, \Phi}$ (we use $<h R . C$ instead of $\leq h R . C$ because it is more "dual" to $\geq h R . C$ )

- if Self $\in \Phi$ then $\exists r$.Self is a concept of $\mathcal{L}_{\Sigma, \Phi}$. 


$$
\begin{gathered}
\top^{\mathcal{I}}=\Delta^{\mathcal{I}} \quad \perp^{\mathcal{I}}=\emptyset \quad(\neg C)^{\mathcal{I}}=\Delta^{\mathcal{I}} \backslash C^{\mathcal{I}} \\
(C \sqcap D)^{\mathcal{I}}=C^{\mathcal{I}} \cap D^{\mathcal{I}} \quad(C \sqcup D)^{\mathcal{I}}=C^{\mathcal{I}} \cup D^{\mathcal{I}} \\
(\exists r . \text { Self })^{\mathcal{I}}=\left\{x \in \Delta^{\mathcal{I}} \mid r^{\mathcal{I}}(x, x)\right\} \\
(\forall R . C)^{\mathcal{I}}=\left\{x \in \Delta^{\mathcal{I}} \mid \forall y\left[R^{\mathcal{I}}(x, y) \Rightarrow C^{\mathcal{I}}(y)\right]\right\} \\
(\exists R . C)^{\mathcal{I}}=\left\{x \in \Delta^{\mathcal{I}} \mid \exists y\left[R^{\mathcal{I}}(x, y) \wedge C^{\mathcal{I}}(y)\right]\right\} \\
(\geq h R . C)^{\mathcal{I}}=\left\{x \in \Delta^{\mathcal{I}} \mid \sharp\left\{y \mid R^{\mathcal{I}}(x, y) \wedge C^{\mathcal{I}}(y)\right\} \geq h\right\} \\
(<h R . C)^{\mathcal{I}}=\left\{x \in \Delta^{\mathcal{I}} \mid \sharp\left\{y \mid R^{\mathcal{I}}(x, y) \wedge C^{\mathcal{I}}(y)\right\}<h\right\}
\end{gathered}
$$

Fig. 1. Interpretation of complex concepts

An interpretation over $\Sigma$ is a pair $\mathcal{I}=\left\langle\Delta^{\mathcal{I}},{ }^{\mathcal{I}}\right\rangle$, where $\Delta^{\mathcal{I}}$ is a non-empty set called the domain of $\mathcal{I}$ and ${ }^{\mathcal{I}}$ is a mapping called the interpretation function of $\mathcal{I}$ that associates each individual $a \in \Sigma_{I}$ with an element $a^{\mathcal{I}} \in \Delta^{\mathcal{I}}$, each concept name $A \in \Sigma_{C}$ with a set $A^{\mathcal{I}} \subseteq \Delta^{\mathcal{I}}$, and each role name $r \in \Sigma_{R}$ with a binary relation $r^{\mathcal{I}} \subseteq \Delta^{\mathcal{I}} \times \Delta^{\mathcal{I}}$. For $r \in \Sigma_{R}$, define $\left(r^{-}\right)^{\mathcal{I}}=\left(r^{\mathcal{I}}\right)^{-1}$. The interpretation function ${ }^{\mathcal{I}}$ is extended to complex concepts as shown in Figure 1, where $\sharp \Gamma$ stands for the cardinality of the set $\Gamma$.

An information system over $\Sigma$ is defined to be a finite interpretation over $\Sigma$. See [10, Examples 19.4-19.6] for examples of information systems in DLs.

A concept $C$ of $\mathcal{L}_{\Sigma, \Phi}$ is satisfiable if there exists an interpretation $\mathcal{I}$ over $\Sigma$ such that $C^{\mathcal{I}} \neq \emptyset$. We say that concepts $C$ and $D$ of $\mathcal{L}_{\Sigma, \Phi}$ are equivalent if $C^{\mathcal{I}}=D^{\mathcal{I}}$ for every interpretation $\mathcal{I}$ over $\Sigma$.

The modal depth of a concept $C$, denoted by $\operatorname{mdepth}(C)$, is defined to be:

- 0 if $C$ is of the form $\top, \perp, A$ or $\exists r$.Self,

- $\operatorname{mdepth}(D)$ if $C$ is of the form $\neg D$,

- $\max \left(\operatorname{mdepth}(D), \operatorname{mdepth}\left(D^{\prime}\right)\right)$ if $C$ is of the form $D \sqcap D^{\prime}$ or $D \sqcup D^{\prime}$,

- mdepth $(D)+1$ if $C$ is of the form $\forall R . D, \exists R . D, \geq h R . C$ or $<h R . C$.

Let $d$ denote a natural number. By $\mathcal{L}_{\Sigma, \Phi, d}$ we denote the sublanguage of $\mathcal{L}_{\Sigma, \Phi}$ that consists of concepts with modal depth not greater than $d$.

\section{Concept Normalization}

There are different normal forms for formulas or concepts (e.g., 9]). We provide below such a form. The aim is to introduce the notion of universal interpretation and a lemma about its existence. Our normal form uses the following normalization rules:

- Replace $\forall R . C$ by $\neg \exists R . \neg C$. Replace $<h R . C$ by $\neg \geq h R . C$.

- Replace $\geq 0$ R.C by $T$.

- Push $\neg$ in depth through $\top, \perp, \neg, \sqcap, \sqcup$ according to De Morgan's laws. 
- Represent $C_{1} \sqcap \ldots \sqcap C_{n}$ as an "and"-set $\sqcap\left\{C_{1}, \ldots, C_{n}\right\}$ to make the order inessential and eliminate duplicates. Use a dual rule for $\sqcup$ and "or" -sets.

- Flatten an "and" -set $\sqcap\left\{\sqcap\left\{C_{1}, \ldots, C_{i}\right\}, C_{i+1}, \ldots, C_{n}\right\}$ to $\sqcap\left\{C_{1}, \ldots, C_{n}\right\}$. Replace $\sqcap\{C\}$ by $C$. Replace $\sqcap\left\{\top, C_{1}, \ldots, C_{n}\right\}$ by $\sqcap\left\{C_{1}, \ldots, C_{n}\right\}$. Replace $\sqcap\left\{\perp, C_{1}, \ldots, C_{n}\right\}$ by $\perp$. Use dual rules for "or" -sets.

- Replace $\exists R . \sqcup\left\{C_{1}, \ldots, C_{n}\right\}$ by $\sqcup\left\{\exists R . C_{1}, \ldots, \exists R . C_{n}\right\}$.

- Replace $\geq h R$. $\sqcup\left\{C_{1}, \ldots, C_{n}\right\}$ by the disjunction (using $\sqcup$ ) of all concepts of the form $\sqcap\left\{\geq h_{1}\right.$ R. $C_{1}, \ldots, \geq h_{n}$ R. $\left.C_{n}\right\}$, where $h_{1}, \ldots, h_{n}$ are natural numbers such that $h_{1}+\cdots+h_{n}=h$.

- Distribute $\sqcap$ over $\sqcup$.

A concept is said to be in the normal form if it cannot be changed by any one of the above rules. The following two lemmas can easily be proved.

Lemma 3.1. Any concept can be transformed to a normal form. If $C^{\prime}$ is the normal form of $C$ then they are equivalent. A concept in the normal form may contain $\sqcup$ only at the most outer level (i.e., either it does not contain $\sqcup$ or it must be of the form $\sqcup\left\{C_{1}, \ldots, C_{n}\right\}$, where $C_{1}, \ldots, C_{n}$ do not contain $\left.\sqcup\right)$.

Lemma 3.2. $\mathcal{L}_{\Sigma, \Phi, d}$ has only finitely many concepts in the normal form. All of them can effectively be constructed.

We say that an interpretation $\mathcal{I}$ over $\Sigma$ is universal w.r.t. a sublanguage of $\mathcal{L}_{\Sigma, \Phi}$ if, for every satisfiable concept $C$ of that sublanguage, $C^{\mathcal{I}} \neq \emptyset$.

Lemma 3.3. There exists a finite universal interpretation w.r.t. $\mathcal{L}_{\Sigma, \Phi, d}$, which can effectively be constructed.

Proof. Let $C_{1}, \ldots, C_{n}$ be all satisfiable concepts in the normal form of $\mathcal{L}_{\Sigma, \Phi, d}$. For each $1 \leq i \leq n$, let $\mathcal{I}_{i}$ be a finite model satisfying $C_{i}$, which can effectively be constructed using some tableau algorithm. Without loss of generality we assume that these interpretations have pairwise disjoint domains. Let $\mathcal{I}$ be any interpretation such that: $\Delta^{\mathcal{I}}=\Delta^{\mathcal{I}_{1}} \cup \ldots \cup \Delta^{\mathcal{I}_{n}} ;$ for $A \in \Sigma_{C}, A^{\mathcal{I}}=A^{\mathcal{I}_{1}} \cup \ldots \cup A^{\mathcal{I}_{n}}$; for $r \in \Sigma_{R}, r^{\mathcal{I}}=r^{\mathcal{I}_{1}} \cup \ldots \cup r^{\mathcal{I}_{n}}$. It is easy to see that $\mathcal{I}$ is finite and universal w.r.t. $\mathcal{L}_{\Sigma, \Phi, d}$.

\section{Bounded Bisimulation for Description Logics}

Indiscernibility in DLs is related to bisimulation. In 4 . Divroodi and Nguyen studied bisimulations for a number of DLs. In [10] Nguyen and Szałas generalized that notion to model indiscernibility of objects and study concept learning. In 116. Tran et al. and Ha et al. generalized that notion further for concept learning. In this section, we present bounded bisimulation for the DLs studied in the current paper in order to investigate C-learnability in those DLs.

Let $d$ be a natural number and let

$-\Sigma$ and $\Sigma^{\dagger}$ be DL-signatures such that $\Sigma^{\dagger} \subseteq \Sigma$

- $\Phi$ and $\Phi^{\dagger}$ be sets of DL-features such that $\Phi^{\dagger} \subseteq \Phi$

- $\mathcal{I}$ and $\mathcal{I}^{\prime}$ be interpretations over $\Sigma$. 
A binary relation $Z_{d} \subseteq \Delta^{\mathcal{I}} \times \Delta^{\mathcal{I}^{\prime}}$ is called an $\mathcal{L}_{\Sigma^{\dagger}, \Phi^{\dagger}, d}$-bisimulation between $\mathcal{I}$ and $\mathcal{I}^{\prime}$ if there exists a sequence of binary relations $Z_{d} \subseteq \cdots \subseteq Z_{0} \subseteq \Delta^{\mathcal{I}} \times \Delta^{\mathcal{I}^{\prime}}$ such that the following conditions hold for every $0 \leq i \leq d, 0 \leq j<d, a \in \Sigma_{I}^{\dagger}$, $A \in \Sigma_{C}^{\dagger}, x, y \in \Delta^{\mathcal{I}}, x^{\prime}, y^{\prime} \in \Delta^{\mathcal{I}^{\prime}}$ and every role $R$ of $\mathcal{L}_{\Sigma^{\dagger}, \Phi^{\dagger}}$ :

$$
\begin{aligned}
& Z_{i}\left(a^{\mathcal{I}}, a^{\mathcal{I}^{\prime}}\right) \\
& Z_{0}\left(x, x^{\prime}\right) \Rightarrow\left[A^{\mathcal{I}}(x) \Leftrightarrow A^{\mathcal{I}^{\prime}}\left(x^{\prime}\right)\right] \\
& {\left[Z_{j+1}\left(x, x^{\prime}\right) \wedge R^{\mathcal{I}}(x, y)\right] \Rightarrow \exists y^{\prime} \in \Delta^{\mathcal{I}^{\prime}}\left[Z_{j}\left(y, y^{\prime}\right) \wedge R^{\mathcal{I}^{\prime}}\left(x^{\prime}, y^{\prime}\right)\right]} \\
& {\left[Z_{j+1}\left(x, x^{\prime}\right) \wedge R^{\mathcal{I}^{\prime}}\left(x^{\prime}, y^{\prime}\right)\right] \Rightarrow \exists y \in \Delta^{\mathcal{I}}\left[Z_{j}\left(y, y^{\prime}\right) \wedge R^{\mathcal{I}}(x, y)\right],}
\end{aligned}
$$

if $Q_{k} \in \Phi^{\dagger}$ and $1 \leq h \leq k$ then

if $Z_{j+1}\left(x, x^{\prime}\right)$ holds and $y_{1}, \ldots, y_{h}$ are pairwise different elements of $\Delta^{\mathcal{I}}$ such that $R^{\mathcal{I}}\left(x, y_{l}\right)$ holds for every $1 \leq l \leq h$ then there exist pairwise different elements $y_{1}^{\prime}, \ldots, y_{h}^{\prime}$ of $\Delta^{\mathcal{I}^{\prime}}$ such that $R^{\mathcal{I}^{\prime}}\left(x^{\prime}, y_{l}^{\prime}\right)$ and $Z_{j}\left(y_{l}, y_{l}^{\prime}\right)$ hold for every $1 \leq l \leq h$

if $Z_{j+1}\left(x, x^{\prime}\right)$ holds and $y_{1}^{\prime}, \ldots, y_{h}^{\prime}$ are pairwise different elements of $\Delta^{\mathcal{I}^{\prime}}$ such that $R^{\mathcal{I}^{\prime}}\left(x^{\prime}, y_{l}^{\prime}\right)$ holds for every $1 \leq l \leq h$ then there exist pairwise different elements $y_{1}, \ldots, y_{h}$ of $\Delta^{\mathcal{I}}$ such that $R^{\mathcal{I}}\left(x, y_{l}\right)$ and $Z_{j}\left(y_{l}, y_{l}^{\prime}\right)$ hold for every $1 \leq l \leq h$,

if Self $\in \Phi^{\dagger}$ then

$$
Z_{0}\left(x, x^{\prime}\right) \Rightarrow\left[r^{\mathcal{I}}(x, x) \Leftrightarrow r^{\mathcal{I}^{\prime}}\left(x^{\prime}, x^{\prime}\right)\right] .
$$

An interpretation $\mathcal{I}$ over $\Sigma$ is finitely branching (or image-finite) w.r.t. $\mathcal{L}_{\Sigma^{\dagger}, \Phi^{\dagger}}$ and $\mathcal{L}_{\Sigma^{\dagger}, \Phi^{\dagger}, d}$ if, for every $x \in \Delta^{\mathcal{I}}$ and every role $R$ of $\mathcal{L}_{\Sigma^{\dagger}, \Phi^{\dagger}}$, the set $\left\{y \in \Delta^{\mathcal{I}} \mid\right.$ $\left.R^{\mathcal{I}}(x, y)\right\}$ is finite.

Let $x \in \Delta^{\mathcal{I}}$ and $x^{\prime} \in \Delta^{\mathcal{I}^{\prime}}$. We say that $x$ is $\mathcal{L}_{\Sigma^{\dagger}, \Phi^{\dagger}, d^{-}}$-equivalent to $x^{\prime}$ if, for every concept $C$ of $\mathcal{L}_{\Sigma^{\dagger}, \Phi^{\dagger}, d}, x \in C^{\mathcal{I}}$ iff $x^{\prime} \in C^{\mathcal{I}^{\prime}}$.

Theorem 4.1 (The Hennessy-Milner Property). Let $d$ be a natural number, $\Sigma$ and $\Sigma^{\dagger}$ be DL-signatures such that $\Sigma^{\dagger} \subseteq \Sigma$, $\Phi$ and $\Phi^{\dagger}$ be sets of $D L$ features such that $\Phi^{\dagger} \subseteq \Phi$. Let $\mathcal{I}$ and $\mathcal{I}^{\prime}$ be interpretations in $\mathcal{L}_{\Sigma, \Phi}$, finitely branching w.r.t. $\mathcal{L}_{\Sigma^{\dagger}, \Phi^{\dagger}}$ and such that for every $a \in \Sigma_{I}^{\dagger}, a^{\mathcal{I}}$ is $\mathcal{L}_{\Sigma^{\dagger}, \Phi^{\dagger}, d^{-}}$-equivalent to $a^{\mathcal{I}^{\prime}}$. Then $x \in \Delta^{\mathcal{I}}$ is $\mathcal{L}_{\Sigma^{\dagger}, \Phi^{\dagger}, d^{-}}$-equivalent to $x^{\prime} \in \Delta^{\mathcal{I}^{\prime}}$ iff there exists an $\mathcal{L}_{\Sigma^{\dagger}, \Phi^{\dagger}, d}$-bisimulation $Z_{d}$ between $\mathcal{I}$ and $\mathcal{I}^{\prime}$ such that $Z_{d}\left(x, x^{\prime}\right)$ holds.

This theorem can be proved analogously to [4, Theorem 4.1].

An $\mathcal{L}_{\Sigma^{\dagger}, \Phi^{\dagger}, d^{-}}$-bisimulation between $\mathcal{I}$ and itself is called an $\mathcal{L}_{\Sigma^{\dagger}, \Phi^{\dagger}, d}$-autobisimulation of $\mathcal{I}$. An $\mathcal{L}_{\Sigma^{\dagger}, \Phi^{\dagger}, d}$-auto-bisimulation of $\mathcal{I}$ is said to be the largest if it is larger than or equal to $(\supseteq)$ any other $\mathcal{L}_{\Sigma^{\dagger}, \Phi^{\dagger}, d}$-auto-bisimulation of $\mathcal{I}$.

Given an interpretation $\mathcal{I}$ over $\Sigma$, by $\sim_{\Sigma^{\dagger}, \Phi^{\dagger}, d, \mathcal{I}}$ we denote the largest

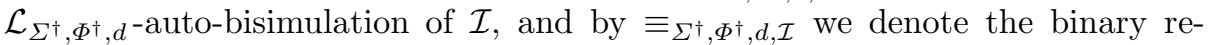
lation on $\Delta^{\mathcal{I}}$ with the property that $x \equiv_{\Sigma^{\dagger}, \Phi^{\dagger}, d, \mathcal{I}} x^{\prime}$ iff $x$ is $\mathcal{L}_{\Sigma^{\dagger}, \Phi^{\dagger}, d^{-}}$-equivalent to $x^{\prime}$. 
Theorem 4.2. Let $d$ be a natural number, $\Sigma$ and $\Sigma^{\dagger}$ be DL-signatures such that $\Sigma^{\dagger} \subseteq \Sigma, \Phi$ and $\Phi^{\dagger}$ be sets of $D L$-features such that $\Phi^{\dagger} \subseteq \Phi$, and $\mathcal{I}$ be an interpretation over $\Sigma$. Then the largest $\mathcal{L}_{\Sigma^{\dagger}, \Phi^{\dagger}, d}$-auto-bisimulation of $\mathcal{I}$ exists and is an equivalence relation. Furthermore, if $\mathcal{I}$ is finitely branching w.r.t. $\mathcal{L}_{\Sigma^{\dagger}, \Phi^{\dagger}}$ then the relation $\equiv_{\Sigma^{\dagger}, \Phi^{\dagger}, d, \mathcal{I}}$ is the largest $\mathcal{L}_{\Sigma^{\dagger}, \Phi^{\dagger}, d}$-auto-bisimulation of $\mathcal{I}$ (i.e. the relations $\equiv_{\Sigma^{\dagger}, \Phi^{\dagger}, d, \mathcal{I}}$ and $\sim_{\Sigma^{\dagger}, \Phi^{\dagger}, d, \mathcal{I}}$ coincide).

This theorem differs from the ones of [10116] in the considered languages. It can be proved analogously to [4, Proposition 5.1 and Theorem 5.2].

We say that a set $Y$ is divided by a set $X$ if $Y \backslash X \neq \emptyset$ and $Y \cap X \neq \emptyset$. Thus, $Y$ is not divided by $X$ if either $Y \subseteq X$ or $Y \cap X=\emptyset$. A partition $P=\left\{Y_{1}, \ldots, Y_{n}\right\}$ is consistent with a set $X$ if, for every $1 \leq i \leq n, Y_{i}$ is not divided by $X$.

Theorem 4.3. Let d be a natural number, $\Sigma$ and $\Sigma^{\dagger}$ be DL-signatures such that $\Sigma^{\dagger} \subseteq \Sigma, \Phi$ and $\Phi^{\dagger}$ be sets of DL-features such that $\Phi^{\dagger} \subseteq \Phi, \mathcal{I}$ be an interpretation over $\Sigma$, and let $X \subseteq \Delta^{\mathcal{I}}$. Then:

1. if there exists a concept $C$ of $\mathcal{L}_{\Sigma^{\dagger}, \Phi^{\dagger}, d}$ such that $X=C^{\mathcal{I}}$ then the partition of $\Delta^{\mathcal{I}}$ by $\sim_{\Sigma^{\dagger}, \Phi^{\dagger}, d, \mathcal{I}}$ is consistent with $X$

2. if the partition of $\Delta^{\mathcal{I}}$ by $\sim_{\Sigma^{\dagger}, \Phi^{\dagger}, d, \mathcal{I}}$ is consistent with $X$ then there exists a concept $C$ of $\mathcal{L}_{\Sigma^{\dagger}, \Phi^{\dagger}, d}$ such that $C^{\mathcal{I}}=X$.

This theorem differs from the ones of 10116 in the considered languages (and the studied class of DLs). It can be proved analogously to [10, Theorem 4].

\section{A Concept Learning Algorithm}

Let $A_{0} \in \Sigma_{C}$ be a concept name standing for the "decision attribute" and suppose that $A_{0}$ can be expressed by a concept $C$ in $\mathcal{L}_{\Sigma^{\dagger}, \Phi^{\dagger}}$, where $\Sigma^{\dagger} \subseteq \Sigma \backslash$ $\left\{A_{0}\right\}$ and $\Phi^{\dagger} \subseteq \Phi$. Let $\mathcal{I}$ be a training information system over $\Sigma$. How can we learn that concept $C$ on the basis of $\mathcal{I}$ ? In 10 Nguyen and Szałas gave a bisimulation-based method for this learning problem. In this section, by adopting a specific strategy we present a modified version of that method, called the MiMoD (minimizing-modal-depth) concept learning algorithm. This algorithm is used for analyzing C-learnability in the next section. It may not give high accuracy for general cases.

Our MiMoD algorithm is as follows:

1. Starting from the partition $\left\{\Delta^{\mathcal{I}}\right\}$, make subsequent granulations to reach a partition consistent with $A_{0}^{\mathcal{I}}$. In the granulation process, we denote the blocks created so far in all steps by $Y_{1}, \ldots, Y_{n}$, where the current partition may consist of only some of them. We do not use the same subscript to denote blocks of different contents (i.e. we always use new subscripts obtained by increasing $n$ for new blocks). We take care that, for each $1 \leq i \leq n, Y_{i}$ is characterized by a concept $C_{i}$ such that $Y_{i}=C_{i}^{\mathcal{I}}$.

2. We use the following concepts as selectors for the granulation process, where $1 \leq i \leq n$ : 
(a) $A$, where $A \in \Sigma_{C}^{\dagger}$

(b) $\exists r$.Self, if Self $\in \Phi^{\dagger}$ and $r \in \Sigma_{R}^{\dagger}$

(c) $\exists r . C_{i}$, where $r \in \Sigma_{R}^{\dagger}$

(d) $\exists r^{-}$. $C_{i}$, if $I \in \Phi^{\dagger}$ and $r \in \Sigma_{R}^{\dagger}$

(e) $\geq h r . C_{i}$, if $Q_{k} \in \Phi^{\dagger}, r \in \Sigma_{R}^{\dagger}$ and $1 \leq h \leq k$

(f) $\geq h r^{-} . C_{i}$, if $\left\{Q_{k}, I\right\} \subseteq \Phi^{\dagger}, r \in \Sigma_{R}^{\dagger}$ and $1 \leq h \leq k$.

A selector $D$ has a higher priority than $D^{\prime}$ if $\operatorname{mdepth}(D)<\operatorname{mdepth}\left(D^{\prime}\right)$.

3. During the granulation process, if

- a block $Y_{i}$ of the current partition is divided by $D^{\mathcal{I}}$, where $D$ is a selector,

- and there do not exist a block $Y_{j}$ of the current partition and a selector $D^{\prime}$ with a higher priority than $D$ such that $Y_{j}$ is divided by $D^{\prime}$

then partition $Y_{i}$ by $D$ as follows:

$-s:=n+1, \quad t:=n+2, n:=n+2$

$-Y_{s}:=Y_{i} \cap D^{\mathcal{I}}, C_{s}:=C_{i} \sqcap D$

- $Y_{t}:=Y_{i} \cap(\neg D)^{\mathcal{I}}, C_{t}:=C_{i} \sqcap \neg D$

- replace $Y_{i}$ in the current partition by $Y_{s}$ and $Y_{t}$.

4. When the current partition becomes consistent with $A_{0}^{\mathcal{I}}$, return $C_{i_{1}} \sqcup \ldots \sqcup C_{i_{j}}$, where $i_{1}, \ldots, i_{j}$ are indices such that $Y_{i_{1}}, \ldots, Y_{i_{j}}$ are all the blocks of the current partition that are subsets of $A_{0}^{\mathcal{I}}$.

Observe that the above algorithm always terminates.

See [10, Examples 19.7 and 19.8] for examples on concept learning in DLs.

Lemma 5.1. Let $\Sigma$ and $\Sigma^{\dagger}$ be DL-signatures such that $\Sigma^{\dagger} \subseteq \Sigma$, $\Phi$ and $\Phi^{\dagger}$ be sets of DL-features such that $\Phi^{\dagger} \subseteq \Phi$, and $\mathcal{I}$ be an interpretation over $\Sigma$. Suppose $A_{0} \in \Sigma_{C} \backslash \Sigma_{C}^{\dagger}$ and $C$ is a concept of $\mathcal{L}_{\Sigma^{\dagger}, \Phi^{\dagger}}$ such that $A_{0}^{\mathcal{I}}=C^{\mathcal{I}}$. Let $C^{\prime}$ be the concept returned by the MiMoD algorithm for $\mathcal{I}$. Then $C^{\prime}$ is a concept of $\mathcal{L}_{\Sigma^{\dagger}, \Phi^{\dagger}}$ such that $C^{\prime \mathcal{I}}=C^{\mathcal{I}}$ and $\operatorname{mdepth}\left(C^{\prime}\right) \leq \operatorname{mdepth}(C)$.

Proof. Clearly, $C^{\mathcal{I}}=A_{0}^{\mathcal{I}}=C^{\mathcal{I}}$. Consider the execution of the MiMoD algorithm on $\mathcal{I}$ that results in $C^{\prime}$. By $\mathcal{P}_{d}$ we denote the partition of $\Delta^{\mathcal{I}}$ at the moment in that execution when $\max \left\{\operatorname{mdepth}\left(C_{i}\right) \mid Y_{i} \in \mathcal{P}_{d}\right\}=d$ and $\mathcal{P}_{d}$ cannot be granulated any more without using some selector with modal depth $d+1$. Let $d_{\max }$ be the maximal value of such a $d$. Let $Z_{d}$ be the equivalence relation corresponding to the partition $\mathcal{P}_{d}$, i.e. $Z_{d}=\left\{\left\langle x, x^{\prime}\right\rangle \mid x, x^{\prime} \in Y_{i}\right.$ for some $Y_{i} \in$ $\left.\mathcal{P}_{d}\right\}$. It is straightforward to prove by induction on $d$ that $Z_{d}$ is an $\mathcal{L}_{\Sigma^{\dagger}, \Phi^{\dagger}, d^{-}}$-autobisimulation of $\mathcal{I}$. Hence, $Z_{d} \subseteq \sim_{\Sigma^{\dagger}, \Phi^{\dagger}, d, \mathcal{I}}$. Since each block of $\mathcal{P}_{d}$ is characterized by a concept of $\mathcal{L}_{\Sigma^{\dagger}, \Phi^{\dagger}, d}, Z_{d}$ is a superset of $\equiv_{\Sigma^{\dagger}, \Phi^{\dagger}, d, \mathcal{I}}$. Since $\equiv_{\Sigma^{\dagger}, \Phi^{\dagger}, d, \mathcal{I}}$ and $\sim_{\Sigma^{\dagger}, \Phi^{\dagger}, d, \mathcal{I}}$ coincide (Theorem 4.2), we have that $Z_{d}=\equiv_{\Sigma^{\dagger}, \Phi^{\dagger}, d, \mathcal{I}}$.

Since the algorithm terminates as soon as the current partition is consistent with $C^{\mathcal{I}}$, it follows that $d_{\max } \leq \operatorname{mdepth}(C)$. Furthermore, if $d_{\max }<\operatorname{mdepth}\left(C^{\prime}\right)$ then we also have $d_{\max }<\operatorname{mdepth}(C)$. Since $\operatorname{mdepth}\left(C^{\prime}\right) \leq d_{\max }+1$, we conclude that $\operatorname{mdepth}\left(C^{\prime}\right) \leq \operatorname{mdepth}(C)$. 


\section{C-Learnability in Description Logics}

Theorem 6.1. Let $d$ be a natural number, $\Sigma$ and $\Sigma^{\dagger}$ be DL-signatures such that $\Sigma^{\dagger} \subseteq \Sigma$, $\Phi$ and $\Phi^{\dagger}$ be sets of DL-features such that $\Phi^{\dagger} \subseteq \Phi$, and $\mathcal{I}$ be a finite universal interpretation w.r.t. $\mathcal{L}_{\Sigma^{\dagger}, \Phi^{\dagger}, d}$. Suppose $A_{0} \in \Sigma_{C} \backslash \Sigma_{C}^{\dagger}$ and $C$ is a concept of $\mathcal{L}_{\Sigma^{\dagger}, \Phi^{\dagger}, d}$ such that $A_{0}^{\mathcal{I}}=C^{\mathcal{I}}$. Then the concept returned by the MiMoD algorithm for $\mathcal{I}$ is equivalent to $C$.

Proof. Let $C^{\prime}$ be the concept returned by the MiMoD algorithm for $\mathcal{I}$. By Lemma 5.1 $C^{\prime \mathcal{I}}=C^{\mathcal{I}}$ and mdepth $\left(C^{\prime}\right) \leq \operatorname{mdepth}(C)$. For the sake of contradiction, suppose $C^{\prime}$ is not equivalent to $C$. Thus, either $C \sqcap \neg C^{\prime}$ or $C^{\prime} \sqcap \neg C$ is satisfiable. Both of them belong to $\mathcal{L}_{\Sigma^{\dagger}, \Phi^{\dagger}, d}$. Since $\mathcal{I}$ is universal w.r.t. $\mathcal{L}_{\Sigma^{\dagger}, \Phi^{\dagger}, d}$, it follows that either $\left(C \sqcap \neg C^{\prime}\right)^{\mathcal{I}}$ or $\left(C^{\prime} \sqcap \neg C\right)^{\mathcal{I}}$ is not empty, which contradicts the fact that $C^{\prime \mathcal{I}}=C^{\mathcal{I}}$.

Theorem 6.2. Any concept $C$ in any description logic that extends $\mathcal{A L C}$ with some features amongst $I, Q_{k}$, Self can be learnt if the training information system is good enough.

Proof. Let the considered logic be $\mathcal{L}_{\Sigma^{\dagger}, \Phi^{\dagger}}$ and let $d=\operatorname{mdepth}(C), \Phi=\Phi^{\dagger}$ and $\Sigma=\Sigma^{\dagger} \cup\left\{A_{0}\right\}$, where $A_{0} \notin \Sigma_{C}^{\dagger}$. By Lemma 3.3. there exists a finite universal interpretation $\mathcal{I}^{\prime}$ w.r.t. $\mathcal{L}_{\Sigma^{\dagger}, \Phi^{\dagger}, d}$. Let $\mathcal{I}$ be the interpretation over $\Sigma$ different from $\mathcal{I}^{\prime}$ only in that $A_{0}^{\mathcal{I}}$ is defined to be $C^{\mathcal{I}^{\prime}}$. Clearly, $\mathcal{I}$ is universal w.r.t. $\mathcal{L}_{\Sigma^{\dagger}, \Phi^{\dagger}, d}$ and $A_{0}^{\mathcal{I}}=C^{\mathcal{I}}$. By Theorem 6.1, the concept returned by the MiMoD algorithm for $\mathcal{I}$ is equivalent to $C$.

\section{Concluding Remarks}

Our Theorem 6.2 given above is a novel interesting result for the concept learning theory in DLs. For this theorem we have introduced universal interpretations and bounded bisimulation in DLs and developed the MiMoD algorithm.

As future work, we intend to study C-learnability in other DLs and for the cases when there is background knowledge like a TBox and/or an RBox.

Acknowledgements. This work was supported in part by Vietnamese Grant No. MOET-B2012-01-24 and by Polish National Science Centre (NCN) under Grant No. 2011/01/B/ST6/02759 as well as by Polish National Centre for Research and Development (NCBiR) under Grant No. SP/I/1/77065/10 by the strategic scientific research and experimental development program: "Interdisciplinary System for Interactive Scientific and Scientific-Technical Information".

\section{References}

1. Baader, F., Calvanese, D., McGuinness, D.L., Nardi, D., Patel-Schneider, P.F. (eds.): Description Logic Handbook. Cambridge University Press (2002)

2. Badea, L., Nienhuys-Cheng, S.-H.: A Refinement Operator for Description Logics. In: Cussens, J., Frisch, A.M. (eds.) ILP 2000. LNCS (LNAI), vol. 1866, pp. 40-59. Springer, Heidelberg (2000) 
3. Cohen, W.W., Hirsh, H.: Learning the Classic description logic: Theoretical and experimental results. In: Proceedings of KR 1994, pp. 121-133 (1994)

4. Divroodi, A.R., Nguyen, L.A.: On bisimulations for description logics. CoRR, abs/1104.1964 (2011); appeared also in Proceedings of CS\&P 2011, pp. 99-110 (2011)

5. Fanizzi, N., d'Amato, C., Esposito, F.: DL-FOIL Concept Learning in Description Logics. In: Železný, F., Lavrač, N. (eds.) ILP 2008. LNCS (LNAI), vol. 5194, pp. 107-121. Springer, Heidelberg (2008)

6. Ha, Q.-T., Hoang, T.-L.-G., Nguyen, L.A., Nguyen, H.S., Szałas, A., Tran, T.-L.: A bisimulation-based method of concept learning for knowledge bases in description logics. Accepted for SoICT 2012 (2012)

7. Iannone, L., Palmisano, I., Fanizzi, N.: An algorithm based on counterfactuals for concept learning in the Semantic Web. Appl. Intell. 26(2), 139-159 (2007)

8. Lehmann, J., Hitzler, P.: Concept learning in description logics using refinement operators. Machine Learning 78(1-2), 203-250 (2010)

9. Nguyen, L.A.: An efficient tableau prover using global caching for the description logic $\mathcal{A L C}$. Fundamenta Informaticae 93(1-3), 273-288 (2009)

10. Nguyen, L.A., Szałas, A.: Logic-Based Roughification. In: Skowron, A., Suraj, Z. (eds.) Rough Sets and Intelligent Systems. ISRL, vol. 42, pp. 517-543. Springer, Heidelberg (2013)

11. Tran, T.-L., Ha, Q.-T., Hoang, T.-L.-G., Nguyen, L.A., Nguyen, H.S., Szałas, A.: Concept learning for description logic-based information systems. Accepted for KSE 2012 (2012)

12. Valiant, L.G.: A theory of the learnable. Commun. ACM 27(11), 1134-1142 (1984) 